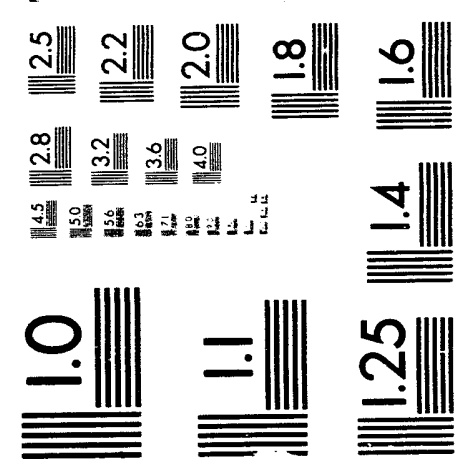



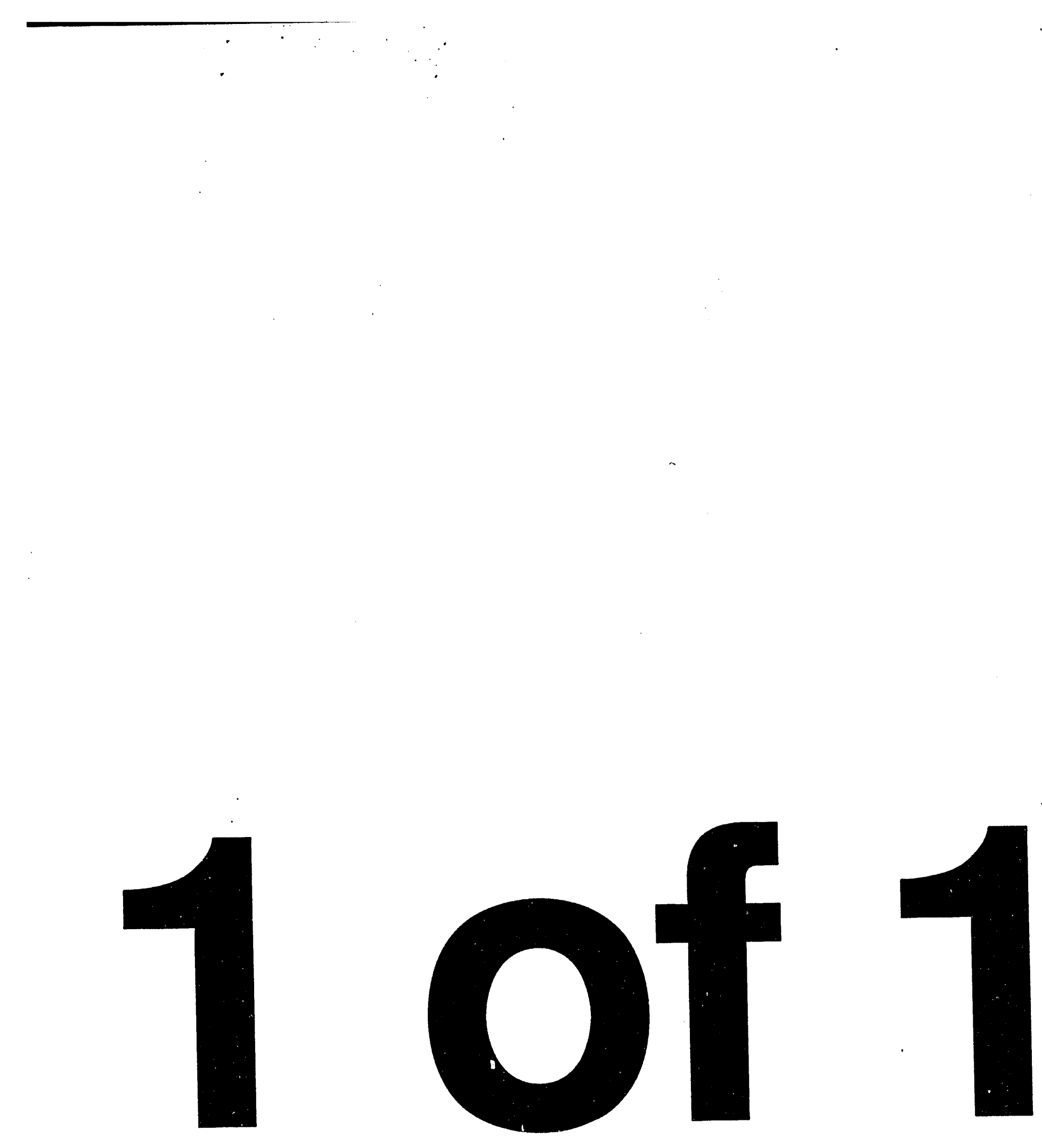
$5.9395(1)$

\title{
On The Application of Allan Variance Method for Ring Laser Gyro Performance Characterization
}

\author{
Lawrence C. Ng
}

October 15, 1993

This is an informal report intended primarily for internal or limited extemal distribution. The opinions and conclusions stated are those of the author and may or may not be those of the Laboratory.

Work performed under the auspices of the U.S. Department of Energy by the Lawrence Livermore National Laboratory under Contract W-7405-Eng-48.

UCRL-ID- 115695 
This document was prepared as an account of work sponsored by an agency of the United States Government. Neither the United States Government nor the University of California nor any of their employees, makes any warranty, express or implied, or assumes any legal liability or responsibility for the accuracy, completeness, or usefulness of any information, apparatus, product, or process disclosed, or represents that its use would not infringe privately owned rights. Reference herein to any specific commercial products, process, or service by trade name, trademark, manufacturer, or otherwise, does not necessarily constitute or imply its endorsement, recommendation, or fa voring by the United States Government or the University of California. The views and opinions of authors expressed herein do not necessarily state or reflect those of the United States Government or the University of California, and shall not be used for advertising or product endorsement purposes.

This report has been reproduced directly from the best available copy.

Available to DOE and DOE contractors from the Office of Scientific and Technical Information P.O. Box 62, Oak Ridge, TN 37831 Prices available from (615) 576-8401, FTS 626.8401

Available to the public from the National Technical Information Service

US. Department of Commerce 5285 Port Royal Rd. Springfield, VA 22161 


\begin{abstract}
This report describes the method of Allan variance and its application to the characterization of a Ring Laser Gyro's (RLG) performance ${ }^{1}$. Allan variance, a time domain analysis technique, is an accepted IEEE standard for gyro specifications. The key attribute of the method is that it allows for a finer characterization and identification of error sources and their contribution to the overall noise statistics.

This report presents an overview of the method, explains the relationship between Allan variance and power spectral density distribution of noise sources, describes the batch and recursive implementation approaches, validates the Allan variance computation with a simulation model, and illustrates the method using data collected from several Honeywell LIMU units.
\end{abstract}

\title{
Administrative Information
}

This work was supported by the RAPTOR/TALON program in O Division; program manager, Nick Colella, and TALON systems engineer, Mark Summers.

1 IEEE Standards Publications 647-1981. IEEE Specification Format Guide and Test Procedure for Single-Axis Laser Gyro. 
Table of Contents

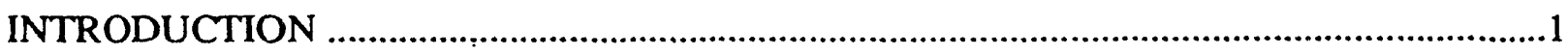

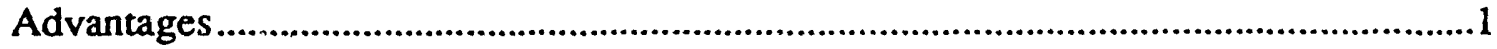

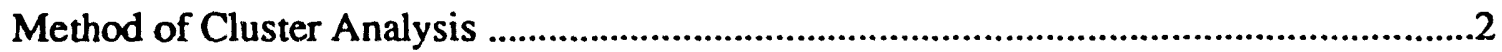

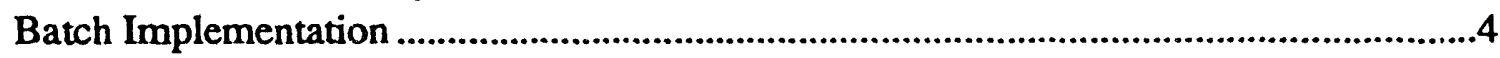

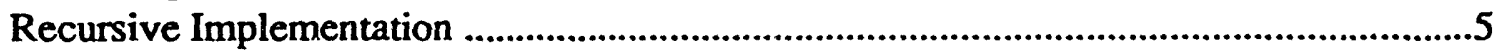

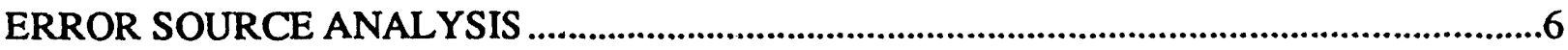

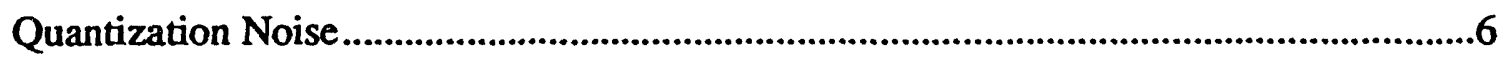

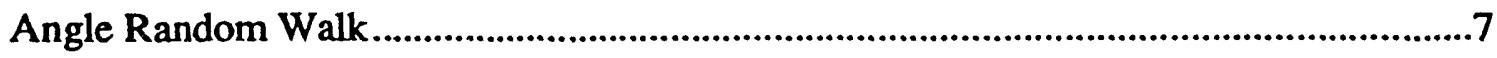

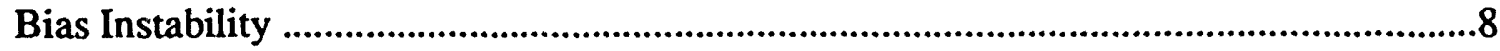

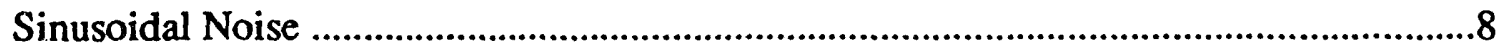

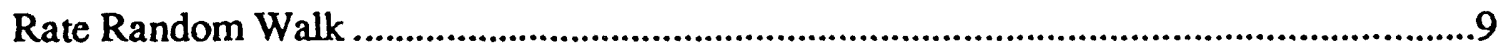

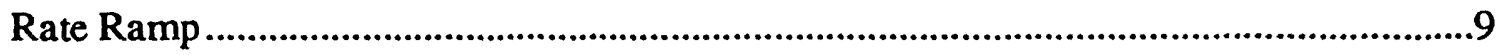

Extraction of Individual Noise Sources from Allan Variance ........................................10

ALLAN VARIANCE ANALYSIS FOR THE HONEYWELL LIMU ................................13

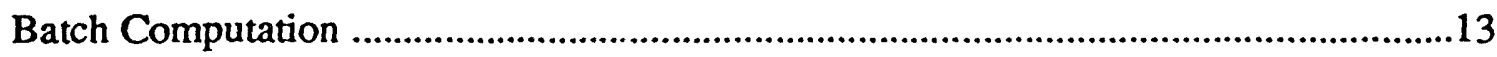

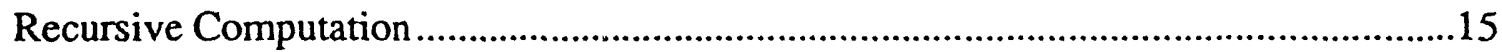

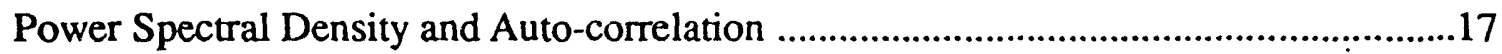

LIMU Performance Under Random Vibration ...................................................19

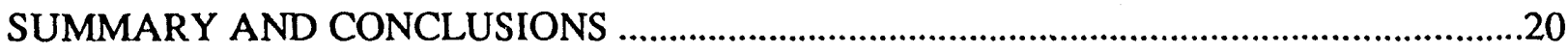

REFERENCES

\section{APPENDICES}

A Derivation of Recursive Formulation of Allan Variance ............................................22

B Derivation of Frequency Domain Equivalence of Allan Variance ...............................24 


\section{List of Figures \& Tables}

Figure 1 Sample Plot of Allan Variance Analysis Results

Figure 2 Example of forming $\mathrm{K}$ clusters from $\mathrm{N}$ data points with $\mathrm{M}$ points per cluster ........ 2

Figure 3 Data Processing Flow Diagram for the Batch Computation Approach ..................... 4

Figure 4 Processing Flow Diagram of a Recursive Implementation of Allan Variance.......... 5

Figure 5 A typical ront Allan variance plot with only quantization, angle random walk, and bias instability

Figure 6 Root Allan variance for $z$-axis of LIMU unit \#5 ............................................. 13

Figure $7 \quad \%$ error in root Allan variance estimateusing one hour of data ............................... 14

Figure 8 Comparison of root Allan variance estimates for $x, y \& z$ axes ............................... 15

Figure 9 Recursive computation of quantization noise .......................................................... 16

Figure 10 Recursive computation of angle random walk ..................................................... 16

Figure 11 Recursive computation of bias instability ......................................................... 16

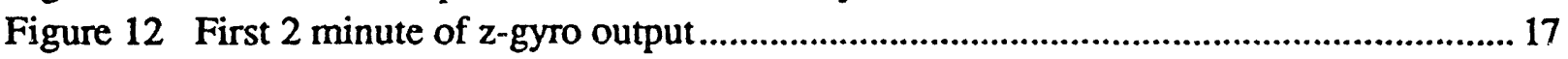

Figure 13 Auto-correlation function of 30 minute z-gyro data ............................................ 18

Figure 14 Power Spectral Density of 30 minute $z$-gyro data ............................................ 18

Figure 15 Angle random walk measured for LIMU unit \#6 z-axis ....................................... 19

Figure 16 Quantization noise measured for LIMU unit \#6 z-axis ....................................... 19

Taüle 1 Summary of Relation Between Allan Variance and Different Error Sources ..........10

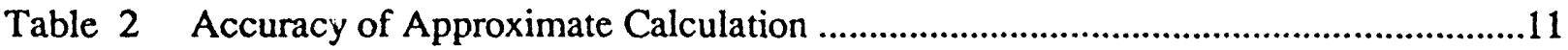

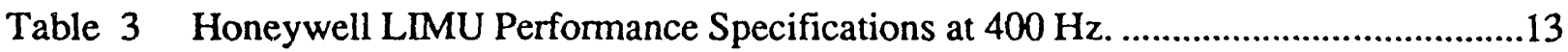

Table 4 Summary of z-axis LIMU unit \#5 performance measures.......................................14

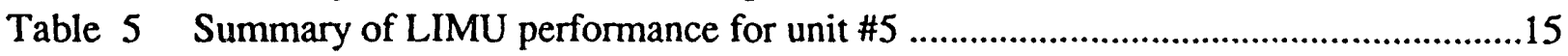




\section{INTRODUCTION}

This report describes the method of Allan variance and its application to the characterization of a Ring Laser Gyro's (RLG) performance [1]. Allan variance, a time domain analysis technique, is an accepted IEEE standard for gyro specifications. The method was initially developed by David Allan of the National Bureau of Standards to quantify the error statistics of a Cesium beam frequency standard employed as the U.S. Frequency Standards in 1960's [2]. The method can, in general, be applied to analyse the error characteristics of any precision measurement instruments. The key attribute of the method is that it allows for a finer, easier characterization and identification of error sources and their contribution to the overall noise statistics.

This report presents an overview of the method, explains the relationship between Allan variance and power spectral density distribution of underlying noise sources, describes the batch and recursive implementation approaches, validates the Allan variance computation with a simulation model, and illustrates the Allan variance method using data collected from several Honeywell LIMU units.

\section{Advantages}

An RLG has many contributing error sources. Predominant error sources included: (1) angle random walk, (2) quantization, (3) bias instability, (4) rate angle walk, (5) rate ramp, and (6) sinusoidal component. The first three error terms are normally included as part of the overall performance specifications. Traditional approaches such as computing the sampled mean and variance from a measurement set do not reveal the underlying error sources. On the other hand, although the computations of autocorrelation function or the power spectral density (PSD) distribution do contain a complete description of the error sources, the results are difficult to interpret or extract ${ }^{2}$.

Allan variance is based on the method of cluster analysis [3]. A data stream is divided into clusters of specified length. The average value of each cluster is computed. A two point sample variance is then calculated from the successive cluster averages. By choosing different cluster length or correlation time for each Allan variance computation, one obtains the Allan variance as a function of correlation time. The attractiveness of this method is that the Allan variance, when plotted in logarithmic scales, can discriminate different contributing etror sources by simply examining the varying slopes on the Allan variance plot. Furthermore, by picking specific values of correlation time, one can easily extract information on angle random walk, quantization noise, bias instability, and etc.

2 A PSD is idcal for identifying cither narrowband hamonic components or broadtband sources, but extracting other contributing components such as bias instability, angle random walk, and quantization is difticult at best. 
Figure 1 shows a sketch of a sample plot of Allan variance results. Note that the error sources of interest have slopes bounded between \pm 1 and that the slopes identify with different contributing sources of gyro noise. The typical correlation time scale for the computation of each component is: several tens of seconds of data for the quantization error, several minutes of data for the angle random walk, several hours of data for the bias instability, and several tens of hours of data for rate random walk and rate ramp. A detailed description of each error source will be given later.

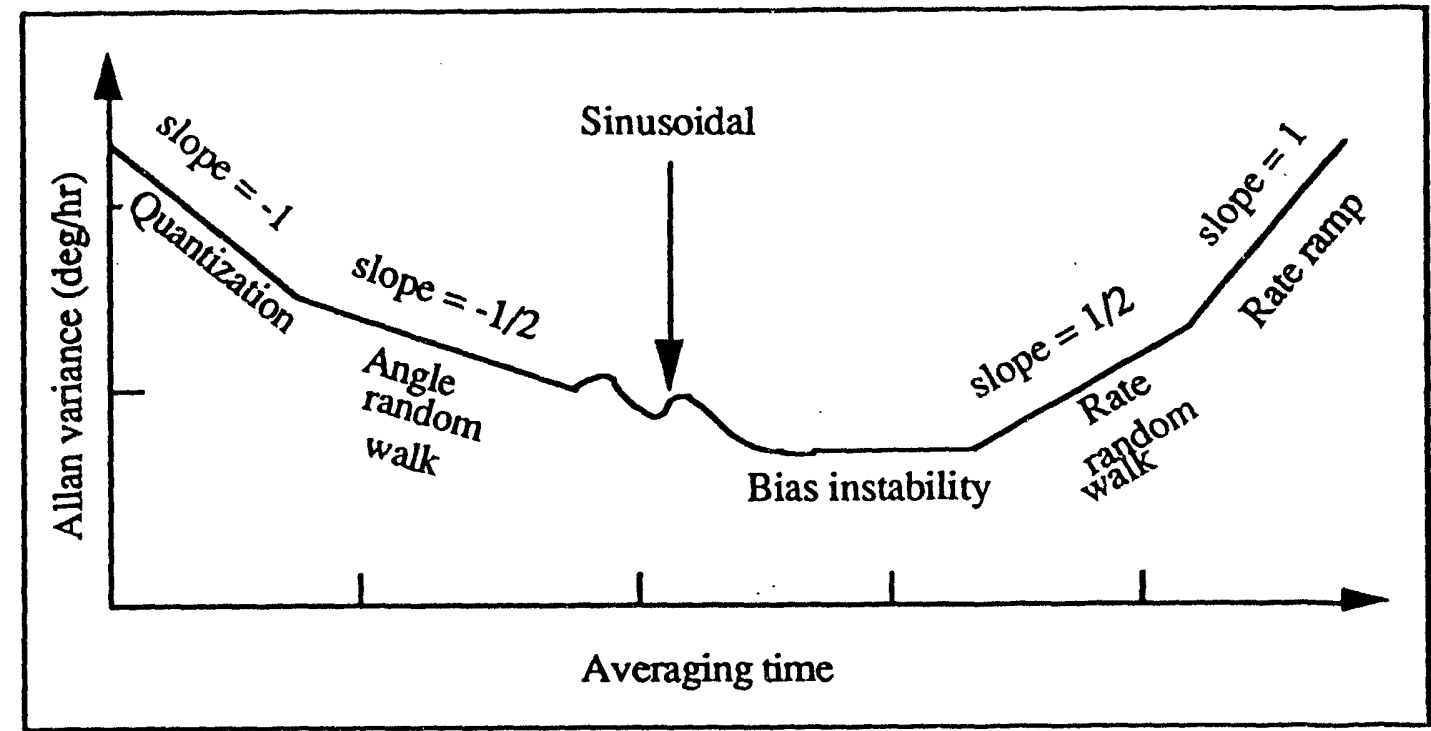

Figure 1 Sample Plot of Allan Variance Analysis Results

\section{Method of Cluster Analysis}

Let angular rate data be taken at a rate of $f_{S}$ samples per second, then from a collection of $\mathrm{N}$ data points, we form $K=N / M$ clusters where $M$ is the number of samples per cluster as illustrated in Figure

$$
\underbrace{\omega_{1}, \omega_{2}, \ldots, \omega_{M}}_{k=1}, \underbrace{\omega_{M+1}, \ldots, \omega_{2 M}}_{k=2}, \ldots, \underbrace{\omega_{N-M}, \ldots, \omega_{N}}_{k=K}
$$

Figure 2. Example of forming $\mathrm{K}$ clusters from $N$ data points with $M$ points per cluster 2. The second step is to compute the average for each cluster from the expression:

$$
\varpi_{k}(M)=\frac{1}{M} \sum_{i=1}^{M} \omega_{(k-1) M+i} \quad ; \quad k=1, \ldots K
$$

The third step is to compute the Allan variance from the cluster averages as follows: 


$$
\begin{aligned}
\sigma_{A}^{2}\left(\tau_{M}\right) & \equiv \frac{1}{2}\left\langle\left(\varpi_{k+1}(M)-\varpi_{k}(M)\right)^{2}\right\rangle \\
& \cong \frac{1}{2(K-1)} \sum_{k=1}^{K-1}\left(\Phi_{k+1}(M)-\varpi_{k}(M)\right)^{2}
\end{aligned}
$$

where \langle\rangle denotes the ensemble average and $\tau_{M}=M / f_{s}$ is the specified correlation time. Note that in Eq.(2) we indicated that the ensemble average is to be computed by the time average. Also note that the expression for the Allan variance comes from the fact that for any two given cluster averages such as $\varpi_{1}$ and $\varpi_{2}$, the two point, unbiased, variance estimate is given by ${ }^{3}$ :

$$
\begin{aligned}
\sigma^{2} & =\left(\varpi_{1}-A V G\right)^{2}+\left(\omega_{2}-A V G\right)^{2} \\
& =\frac{1}{2}\left(\omega_{2}-\varpi_{1}\right)^{2}
\end{aligned}
$$

where $A V G=\left(\varpi_{1}+\varpi_{2}\right) / 2$ is the two sample average. The accuracy in the estimate of the root Allan variance increases with additional number of cluster averages. In general the 1- $\sigma$ accuracy of the computation for $\mathrm{K}$ cluster averages is given by:

$$
\text { \%error }=\frac{100}{\sqrt{2(K-1)}}
$$

The computation of Allan variance from Eqs. (1) and (2) can be implemented in either a batch mode or a recursive mode. The advantage for the batch mode is that one can process a large chunk of data at a time and take advantage of vectorized computation for speed. However, the batch approach requires the availability of a large storage device. For example, the Honeywell LIMU that we are currently testing at LLNL requires a storage capability of $6 \mathrm{MB}$ per channel per hour and there are six channels of output of interest. On the other hand, a recursive implementation can be executed in real time and requires minimum capability for data storage if the number of cluster channels is small. This report describes both approaches. The batch approach is more efficient for post-testing data analysis whereas the recursive approach is needed for real time processing of experimental data -- expecially for extracting error source parameters which require several hours of IMU data.

3

$$
\sigma^{2}=\left[\left(\omega_{1}-\varpi\right)^{2}+\left(\omega_{2}-\varpi\right)^{2}+L+\left(\omega_{N}-\varpi\right)^{2}\right] /(N-1), \text { Papoulis, p246, McGraw-Hill, } 1965
$$




\section{Batch Implementation}

The batch implementation is very much followed the description given in Eqs. (1) and (2). Figure 3 provides a pictorial description of the underlying approach. However an actual implementation may require segmenting the data into smaller data sets because of limitation of computer array size. When this happens additional work will be needed to piece the segmented results together.

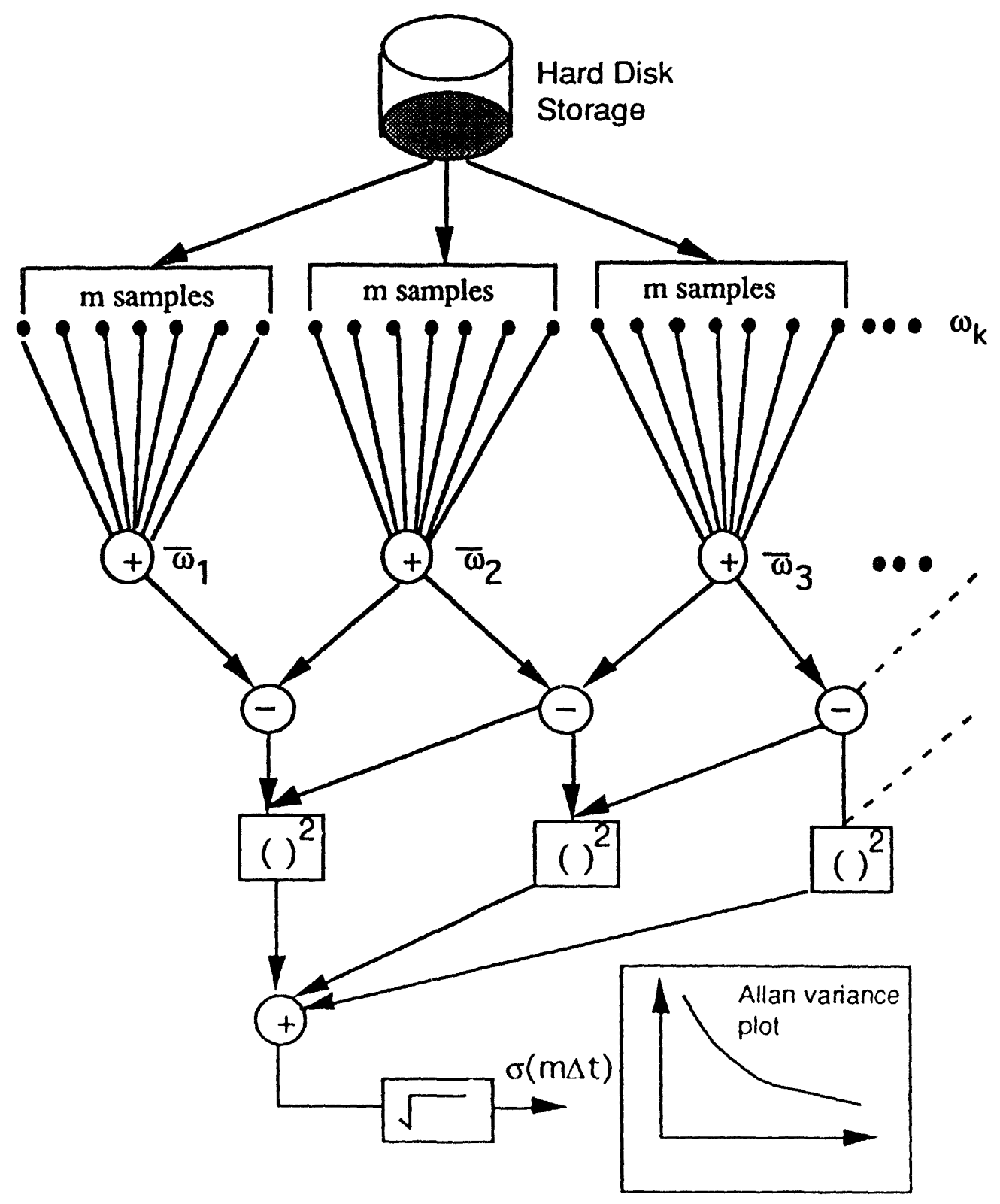

Figure 3 Data Processing Flow Diagram for the Batch Computation Approach 


\section{Recursive Implementation}

A recursive implementation requires recasting Eqs. (1) \& (2) into a recursive form. The resulting two recursion equations are called respectively rate recursion and clusters recursion and are summarized respectively in Eqs. (5) \& (6). Note that the actual derivation is given in Appendix A. For the kth cluster of length $M$, the recursive formulation of (1) can be written as:

$$
\varpi_{k}(m)=\left[\frac{m-1}{m}\right] \varpi_{k}(m-1)+\left[\frac{1}{m}\right] \omega_{(k-1) M+m} \quad ; m=1, \cdots M, k=1, \cdots K .
$$

Also for the kth cluster and correlation length $\mathrm{m}$, the recursive formulation of (2) can be written as:

$$
\sigma_{k}^{2}(m)=\left[\frac{k-2}{k-1}\right] \sigma_{k-1}^{2}(m)+\left[\frac{1}{2(k-1)}\right]\left(\varpi_{k}-\varpi_{k-1}\right) ; m=1, \cdots M, k=1,2, \cdots
$$

A correspondent processing flow diagram is given in Figure 4. Note that for each rate measurement, the average rate is computed sequentially. When the cumulative average rate reaches correlation length $m$, the $m$ th cluster is updated accordingly. The square root of each cluster recursion output yields the root Allan variance.

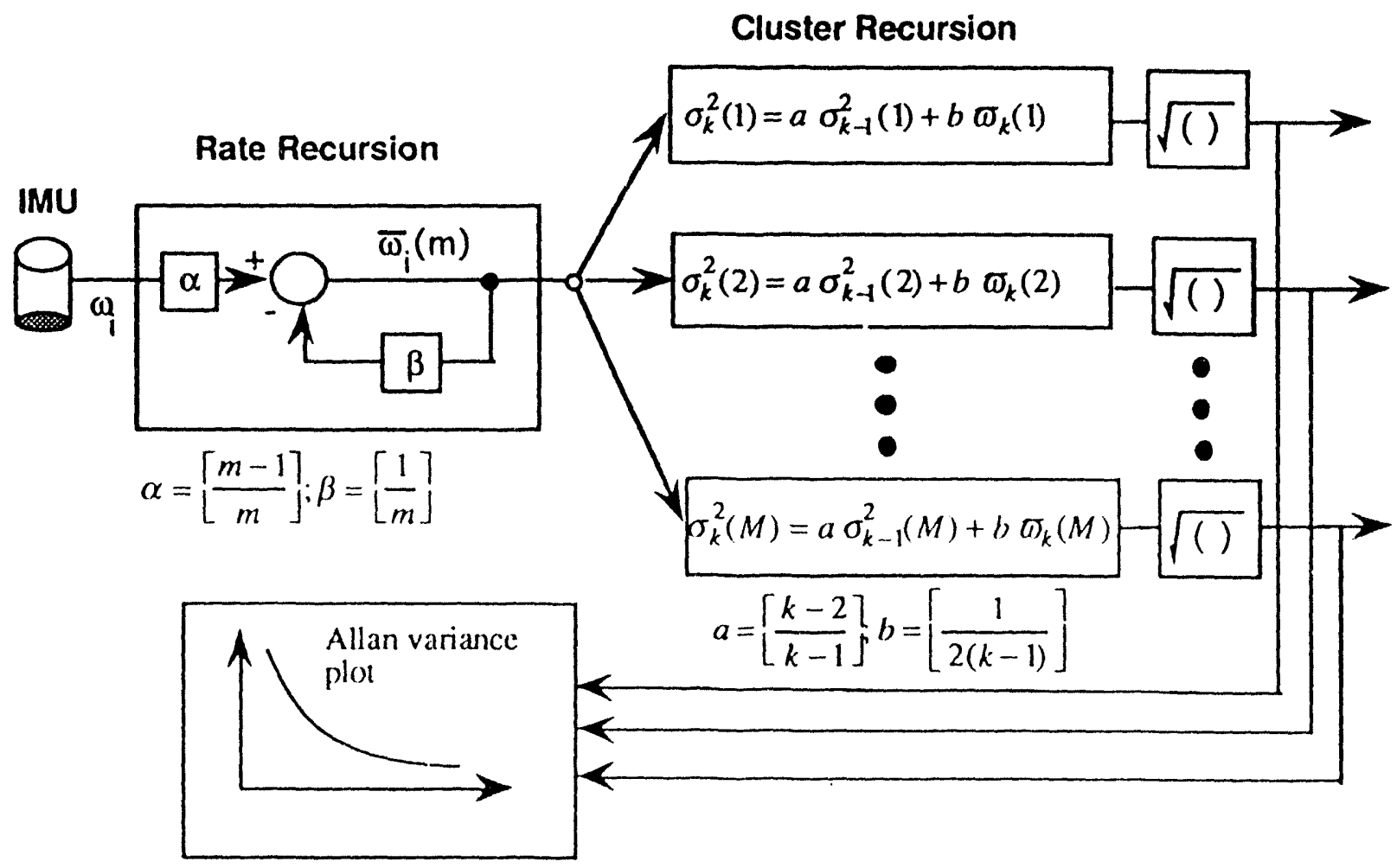

Figure 4 Processing Flow Diagram of a Recursive Implementation of Allan Variance 


\section{ERROR SOURCE ANALYSIS}

As was shown in Figure 1, the most attractive feature of Allan variance is its ability to sort out various error components by the slopes on the root Allan variance plot. In this Section we will further clarify the relation of Allan variance and noise source characterization. The key for developing these important relations is found in the relation of Allan variance expressed in the frequency domain. The relation was presented in Appendix B and summarized as follow:

$$
\sigma^{2}(\tau)=\frac{1}{2}\left\langle\varpi_{k+1}-\varpi_{k}\right\rangle^{2}=4 \int_{0}^{-} d f S_{\omega}(f) \frac{\sin ^{4}(\pi f \tau)}{(\pi f \tau)^{2}}
$$

where $\tau$ is the cluster correlation time, and $S_{\omega}(f)$ is the Power Spectral Density (PSD) of an IMU's measured rate information. Using (7) we can show that the computed Allan variance of different error sources can be expressed in various powers of correlation time.

\section{Quantization Noise}

This noise is strictly due to the discrete/quantized nature of a sensor's output. The readout electronics of a device are in terms of counts ${ }^{4}$. The quantization noise represents the minimum resolution level of the sensor. The PSD for such a process, given in reference 4 , is

$$
\begin{aligned}
S_{\theta}(f) & =\tau Q^{2}\left(\frac{\sin (\pi f \tau)}{\pi f \tau}\right)^{2} \\
& =\tau Q^{2} ; \text { for } f<\frac{1}{2 \tau} .
\end{aligned}
$$

where $Q$ is the quantization noise coefficient. Its theoretical limit is equal to $S / \sqrt{12}$, where $S$ is the gyro scale factor, for tests with fixed and uniform sampling times. The rate PSD is related to the angle PSD (by a differentiation process) through the equation:

$$
S_{\omega}(f)=(2 \pi f)^{2} S_{\theta}(f)
$$

and is

$$
\begin{aligned}
S_{\omega}(f) & =\frac{4 Q^{2}}{\tau} \sin ^{2}(\pi f \tau) \\
& =(2 \pi f)^{2} Q^{2} \tau \quad ; \text { for } \quad f<\frac{1}{2 \tau}
\end{aligned}
$$

Substitution of (10) in (7) and performing the integration yields:

\footnotetext{
${ }^{4}$ For example, the Honeywell LIMU's output is one $\mu \mathrm{rad} / \mathrm{count}$ in angular rate and $4 \mathrm{mg} / \mathrm{count}$ in acceleration.
} 


$$
\sigma_{Q}^{2}(\tau)=\frac{3 Q^{2}}{\tau^{2}}
$$

Therefore the root Allan variance of the quantization noise when plotted in a log-log scale is represented by a slope of -1 . Note that the root Allan variance is normally expressed in units of $\mathrm{deg} /$ hour. Thus the quantization noise level can be evaluated at any point on the root Allan variance plot where the slope is -1 . Also note that quantization noise has a short correlation time or equivalently a wide bandwidth. Since wideband noise can usually be filtered out because of low bandwidth of the vehicle motion, it is not z. major source of error of concerns for RLG.

\section{Angle Random Walk}

Angle random walk is a result of integrating a wideband rate PSD noise [4]. Angle random walk is a major source of error for RLGs that employ randomized dither as anti-lock mechanism [5]. Angle random walk noise is typified by a small varying drift with increasing variance as a function of time. Angle random walk noise typically has a bandwidth less than 10 $\mathrm{Hz}$ and is therefore within the bandwidth of most attitude control systems. Therefore angle random walk is a major source of error that limits the performance of an attitude control system.

When the input angular rate is snaall $(\leq 0.2 \% \mathrm{sec})$, an RLG with two counter-rotation beams would experience a lock-up phenomenon (ie., faileci to detect non-zero input rate). To avoid this problem, one approach is to dither the RLG assembly with a sinusoidal motion. Furthermore, to avoid the build up of angular error during each zero rate crossing, the dither amplitude for each half cycle is randomized. The random zero crossing of the dither signal generates a wideband noise in the rate power spectrum. The associated PSD of the wideband rate noise can be represented by:

$$
S_{\omega}(f)=N^{2}
$$

where $\mathrm{N}$ is the angle random walk coefficient usually expressed in $\mathrm{deg} / \mathrm{hour} / \mathrm{VHz}$. Substitution of (12) into (7) and performing the integration yields:

$$
\sigma_{a r w}^{2}(\tau)=\frac{N^{2}}{\tau} .
$$

Note that Eq.(13) indicates that a $\log -\log$ plot of root Allan variance versus $\tau$ has a slope of $-1 / 2$. Furthermore the numerical value of $\mathrm{N}$ can be obtained directly by reading the slope line at $\tau=1$ hour. 


\section{Bias Instability}

This is the low frequency bias fluctuations in the measured rate data. The origin of this noise is in the RLG discharge assembly, the electronics, or other components susceptible to random "flickering". The rate PSD associated with this noise, also known as $1 / \mathrm{f}$ noise [6] is:

$$
S_{\omega}(f)=\left\{\begin{array}{cc}
\left(\frac{B^{2}}{2 \pi}\right) \frac{1}{f} & ; f \leq f_{o} \\
0 & ; f>f_{o}
\end{array}\right.
$$

where $B$ is the bias instability coefficient and $f_{0}$ is the $3 \mathrm{~dB}$ cutoff frequency. Again substitution of (14) in (7) and performing the integration yields:

$$
\begin{aligned}
\sigma_{b}^{2}(\tau) & =\frac{2 B^{2}}{\pi}\left[\begin{array}{c}
\ln 2-\frac{\sin ^{3}\left(\pi f_{0} \tau\right)}{2\left(\pi f_{0} \tau\right)^{2}}\left(\sin \left(\pi f_{0} \tau\right)+4 \pi f_{0} \tau \cos \left(\pi f_{0} \tau\right)\right. \\
+C_{i}\left(2 \pi f_{0} \tau\right)-C_{i}\left(4 \pi f_{0} \tau\right)
\end{array}\right] \\
& =\left(\frac{B}{0.6648}\right)^{2} \quad ; \quad \text { for } \tau>>\frac{1}{f_{0}} .
\end{aligned}
$$

where $\mathrm{C}_{\mathrm{i}}(\mathrm{)}$ ) is the cosine-integral function. Thus the bias instability value can be read off the root Allan variance plot at the region where the slope is zero.

\section{Sinusoidal Noise}

The PSD of this noise is characterized by a number of distinct frequencies. High frequency noise may originate from plasma oscillations in the laser discharge [7, 8]. A low frequency source could be the slow motion of the test platform due to periodic environmental changes. A representation of the $\mathrm{PSD}$ of this noise containing a single frequency is given as:

$$
S_{\omega}(f)=\frac{1}{2} \omega_{o}^{2}\left[\delta\left(f-f_{o}\right)+\delta\left(f+f_{o}\right)\right]
$$

where $\omega_{0}$ is the rate amplitude, $f_{O}$ is the frequency and $\delta()$ is the Dirac delta function. Substitution of (17) in (7) and performing the integration yie!ds:

$$
\sigma_{s}^{2}(\tau)=\omega_{o}^{2}\left(\frac{\sin ^{2} \pi f_{o} \tau}{\pi f_{o} \tau}\right)^{2}
$$


Thus the root Allan variance of a sinusoid when plotted in $\log -\log$ scale would indicate sinusoidal behavior with successive peaks attentuated at a slope of -1 . This is one case where a conventional PSD plot is superior in identifying the sinusoidal components.

\section{Rate Random Walk}

This noise is a result of integrating a wideband acceleration PSD. This is a random process of uncertain origin, possibly a limiting case of an exponentially correlated noise with a very long correlation time. The mechanical gyro as well as rate biased laser gyros exhibit this noise term. The rate PSD associated with this noise is (from integrating a wideband acceleration spectrum):

$$
S_{\omega}(f)=\left(\frac{K}{2 \pi}\right)^{2} \frac{1}{f^{2}}
$$

where $K$ is the rate random walk coefficient. Substitution of (18) in (7) and performing the integration yields:

$$
\sigma_{m w}^{2}(f)=\left(\frac{K^{2}}{3}\right) \tau
$$

This indicates that Rate Random Walk is represented by a slope of $+1 / 2$ on a log-log plot of $\sigma(\tau)$ versus $\tau$. The unit of $\mathrm{K}$ is usually given in $\operatorname{deg} / \mathrm{hr}^{2} / \sqrt{\mathrm{Hz}}$.

\section{Rate Ramp}

This is more of a deterministic error rather than a random noise. Its presence in the data may indicate a very slow monotonic change of the RLG intensity persisting over a long period of time. It could also be due to a very small acceleration of the platform in the same direction and persisting over a long period of time ( $\sim$ hours). It appears as a genuine input to the RLG given by:

$$
\omega(t)=R t
$$

where $\mathrm{R}$ is the rate ramp coefficient. By forming and operating on the clusters of data containing an input given by (20), we obtain:

$$
\sigma_{r r}^{2}(\tau)=\frac{R^{2} \tau^{2}}{2}
$$

This indicates that the rate ramp noise has slope of +1 in the $\log -\log$ plot of $\sigma(\tau)$ versus $\tau$. 


\section{Extraction of Individual Noise Sources from Allan Variance}

In general, any number of random noise components may be present in the data depending on the type of device and the environment in which the data is obtained. If the noise sources are statistically independent, then the computed Allan variance is sum of the squares of each error type. A method to extract the level of contribution for each component is needed. Relationships of various error sources and Allan variance as discussed above is summarized in Table 1.

\begin{tabular}{|l|c|c|c|}
\hline \multicolumn{1}{|c|}{ Noise Types } & Parameter of interest & Units & $\begin{array}{c}\text { Root Allan Variance } \\
\text { (deg/hr) }\end{array}$ \\
\hline \hline Quantization & $\mathrm{Q}$ & $\mu \mathrm{rad}$ & $\sigma_{Q}=\frac{\sqrt{3} Q}{\tau}$ \\
\hline Angle Random Walk & $\mathrm{N}$ & $\mathrm{deg} / \mathrm{hhr}$ & $\sigma_{\text {an }}=\frac{N}{\sqrt{\tau}}$ \\
\hline Bias Instability & $\mathrm{B}$ & $\mathrm{deg} / \mathrm{hr}$ & $\sigma_{\mathrm{b}}=\frac{B}{0.6648}$ \\
\hline Sinusoidal & $\omega_{\mathrm{o}}$ & $\mathrm{deg} / \mathrm{hr}$ & $\sigma_{s}=\omega_{o}\left(\frac{\sin ^{2}\left(\pi f_{0} \tau\right)}{\pi f_{0} \tau}\right)$ \\
\hline Rate Random Walk & $\mathrm{K}$ & $(\mathrm{deg} / \mathrm{hr}) / \sqrt{\mathrm{hr}}$ & $\sigma_{m}=K \sqrt{\frac{\tau}{3}}$ \\
\hline Rate Ramp & $\mathrm{R}$ & $(\mathrm{deg} / \mathrm{hr} / \mathrm{hr})$ & $\sigma_{r}=\frac{R \tau}{\sqrt{2}}$ \\
\hline
\end{tabular}

Table 1 Summary of Relation Between Allan Variance and different Error Sources

Therefore, assuming statistically independent error sources, the Allan variance can be expressed as composing one or more error sources as follows:

$$
\sigma_{\text {total }}^{2}=\sigma_{Q}^{2}+\sigma_{a r w}^{2}+\sigma_{\text {bias }}^{2}+\sigma_{\text {sin usoid }}^{2}+\sigma_{r r w}^{2}+\sigma_{r}^{2}
$$

and the root Allan variance is given by:

$$
\begin{aligned}
\sigma_{A}(\tau) & =\sqrt{\sigma_{\text {cotal }}^{2}} \\
& =f\left(\sigma_{Q}, \sigma_{a r w}, \sigma_{b}, \sigma_{\text {sin }}, \sigma_{r m}, \sigma_{r r}\right) \\
& =\sum_{n=-2}^{2} A_{n} \tau^{n / 2}
\end{aligned}
$$

where the coefficients $A_{n}$ are obtained in a least mean squares sense. 
Assuming that the computed root Allan variance is expressed in deg/hr, and $\tau$ is in seconds, then the following expressions can be used for the $1-\sigma$ measure of quantization noise, angle random walk, and bias instability respectively:

$$
\begin{aligned}
& Q=\left(\frac{\pi * 10^{6}}{180 * 3600 * \sqrt{3}}\right) A_{-2}(\mu \mathrm{rad}) \\
& N=\left(\frac{A_{-1}}{60}\right)(\mathrm{deg} / \mathrm{hr}) \\
& B=(0.6648) A_{o}(\mathrm{deg})
\end{aligned}
$$

In many instances, and especially for real time data analysis, a quick approximation which provides an upper bound on the predicted performance can be obtained without using the least mean squares fit approach. This is doable because the noise components tend to dominate at different correlation time intervals. For example, let the triplet $\{\tau q, \tau n, \tau b\}$ represent the time on the root Allan variance versus $\tau$ plot where the parameters $Q, N$, and $B$ are to be evaluated. Then the expression that can quickly bound the values of these parameters are:

$$
\begin{aligned}
& Q=\left(\frac{\pi * 10^{6}}{180 * 3600 * \sqrt{3}}\right) \sigma_{\Lambda}\left(\tau_{q}\right) \tau_{q}(\mu \mathrm{rad}) \\
& N=\left(\frac{\sigma_{\Lambda}\left(\tau_{n}\right) \sqrt{\tau_{n}}}{60}\right)(\mathrm{deg} / h r) \\
& B=(0.6648) \sigma_{\Lambda}\left(\tau_{b}\right)
\end{aligned}
$$

Assuming a typical set of parameters for the RLG, (say, $Q=10 \mu \mathrm{rad}, \mathrm{N}=0.1 \mathrm{deg} / \mathrm{Vhr}$, and $\mathrm{B}=1 \mathrm{deg}$. ), a corresponding root Allan variance plot is shown in Figure 5 obtained from solving for the coefficients $A_{k}$ for $k=$ $\{-2,-1,0\}$ as given in Eq. (24). Note that we have neglected to include all other noise

\begin{tabular}{|c|c|c|c|}
\hline $\begin{array}{c}\text { Error } \\
\text { Type }\end{array}$ & $\begin{array}{c}\text { True } \\
\text { Value }\end{array}$ & $\begin{array}{c}\text { Estimated } \\
\text { Bound }\end{array}$ & Error \\
\hline \hline $\mathrm{Q}$ & $10 \mu \mathrm{rad}$ & $11.72 \mu \mathrm{rad}$ & $17 \%$ \\
\hline $\mathrm{N}$ & $0.1 \mathrm{deg} / \mathrm{hr}$ & $0.18 \mathrm{deg} / \mathrm{hhr}$ & $80 \%$ \\
\hline $\mathrm{B}$ & $1 \mathrm{deg} / \mathrm{hr}$ & $1.4 \mathrm{deg} / \mathrm{hr}$ & $40 \%$ \\
\hline
\end{tabular}

Table 2 Accuracy of Approximate Calculation components except that of quantization, angle random walk, and bias instability. Also note that an alternate calculation approach developed by A. Morgan of Honeywell [9] assumed the underlying noise model included only angle random walk. Table 2 summarizes the accuracy in using the approximated expressions as given in Eq. (25). For the typical set of parameters we have chosen for the RLG, the approximate calculations bound the quantization noise by $20 \%$, angle random walk by $80 \%$, and bias instability by $40 \%$. Thus for a more precise estimate of an RLG performance, a complete root Allan variance versus correlation time plot should be generated (may take several hours of actual 
data recording). Then appropriate noise components can be identified by measuring varying slopes of the plot. A least mean squares fit procedure may then be applied to extract the various components.

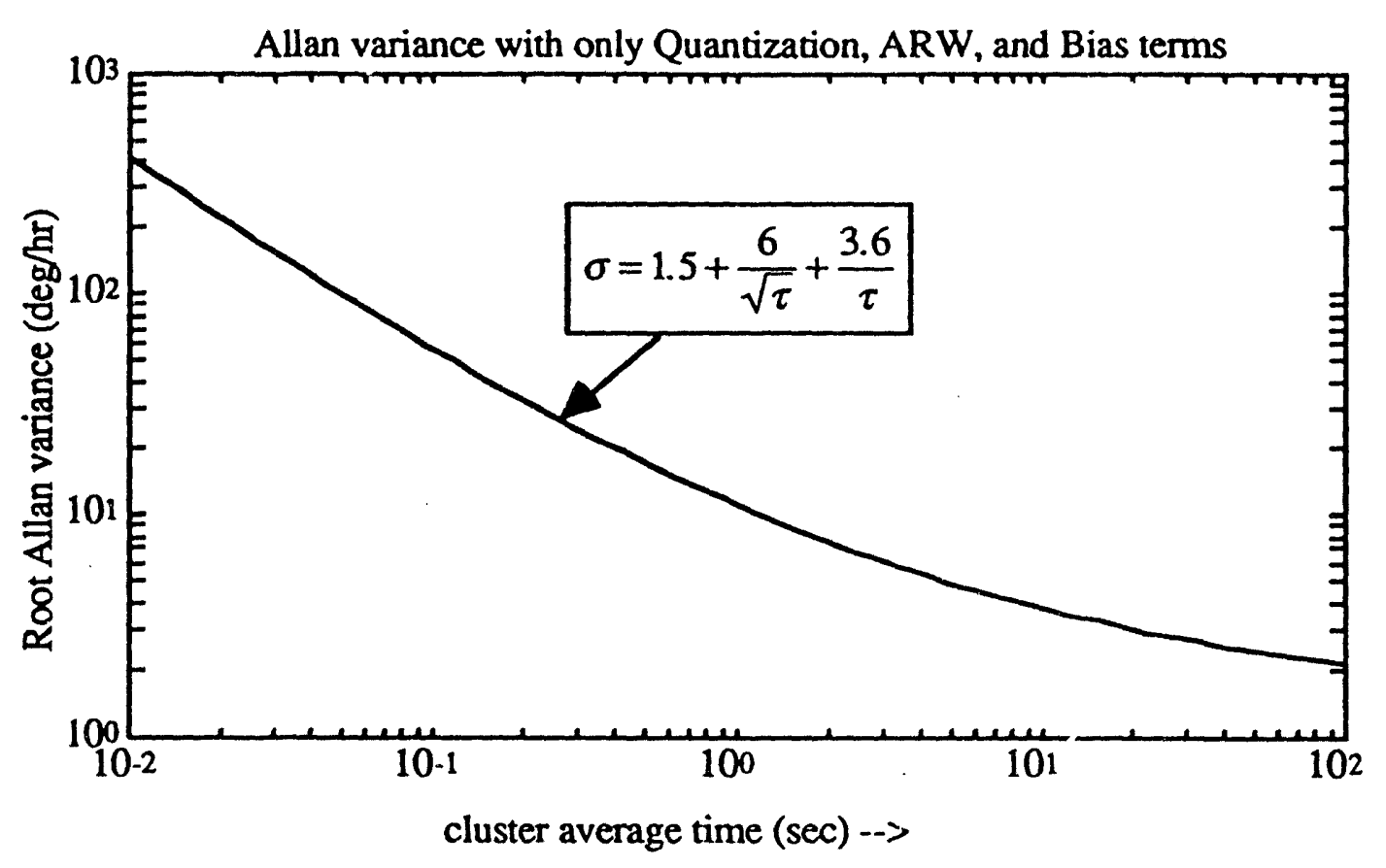

Figure 5 A typical root Allan variance plot with only quantization, angle random walk, and bias instability. 


\section{ALLAN VARIANCE ANALYSIS FOR THE HONEYWELL LIMU}

LLNL took delivery of seven RLG units of Lightweight ( $450 \mathrm{gm})$ IMU (LIMU) manufactured by Honeywell recently. These units all employed a dithering mechansim as a low rate anti-lock device. Thus angle random walk will be the predominant noise source affecting the performance of the attitude pointing and guidance loops. The advertised specification is given in Table 3.

\begin{tabular}{|l|l|}
\hline Gyro noise (readout or quantization) & $7.5 \mu \mathrm{rad}$ \\
\hline Gyro angle random walk & $0.125 \mathrm{deg} / \mathrm{hr}$ \\
\hline Gyro bias instability & $1 \mathrm{deg} / \mathrm{hr}$ \\
\hline Gyro scale factor & $0.025 \%$ \\
\hline & \\
\hline Accel noise (readout) & $40 \mathrm{mg}$ \\
\hline Accel quantization (LSB) & $4 \mathrm{mg}$ \\
\hline Accel bias & $3 \mathrm{mg}$ \\
\hline Accel scale factor & $0.035 \%$ \\
\hline
\end{tabular}

Table 3 Honeywell LIMU Performance Specifications at $400 \mathrm{~Hz}$.

\section{Batch Computation}

A one hour sample of LIMU unit \#5 stationary data was selected for analysis. Note that our approach here is not to provide an extensive performance analysis of the Honeywell LIMU, but instead, we want to demonstrate the use of root Allan variance and the extraction of error components. Figure 6 shows the root Allan variance for the $\mathrm{z}$-axis gyro of LIMU unit \#5.

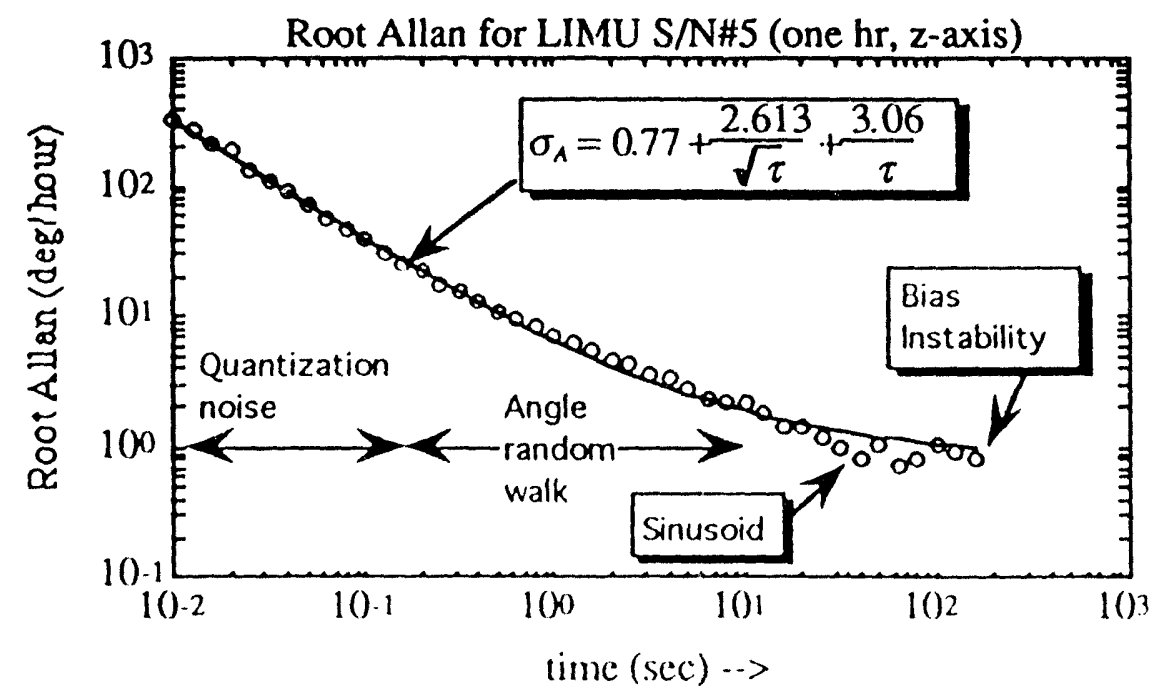

Figure 6 Root Allan variance for z-axis of LIMU unit \#5 
The root Allan variance plot was least mean squares fitted for quantization noise, angle random walk, and bias instability. The fit was quite good. Table 4 summarizes the least mean square fitted results along with the upper bound calculations. It shows that the LIMU meets the design specification in quantization, angle random walk, and bias instability.

\begin{tabular}{|l|l|l|l|}
\hline & LIMU Specification & Least squares fit & Upper bound Calculation \\
\hline Quantization & $7.5 \mu \mathrm{rad}$ & $8.6 \mu \mathrm{rad}$ & $8 \mu \mathrm{rad}$ \\
\hline Angle random walk & $0.125 \mathrm{deg} / \mathrm{hr}$ & $0.04 \mathrm{deg} / \mathrm{hr}$ & $0.125 \mathrm{deg} / \mathrm{hr}$ \\
\hline Bias Instability & $1 \mathrm{deg} / \mathrm{hr}$ & $0.52 \mathrm{deg} / \mathrm{hr}$ & $1 \mathrm{deg} / \mathrm{hr}$ \\
\hline
\end{tabular}

Table 4 Summary of $z$-axis LIMU unit \#5 performance measures

Figure 7 shows the accuracy in the estimate of root Allan variance (RAV) and their extracted parameters. Note that even for a one hour data record, the accuracy in estimating the bias instability term is only about $10 \%$.

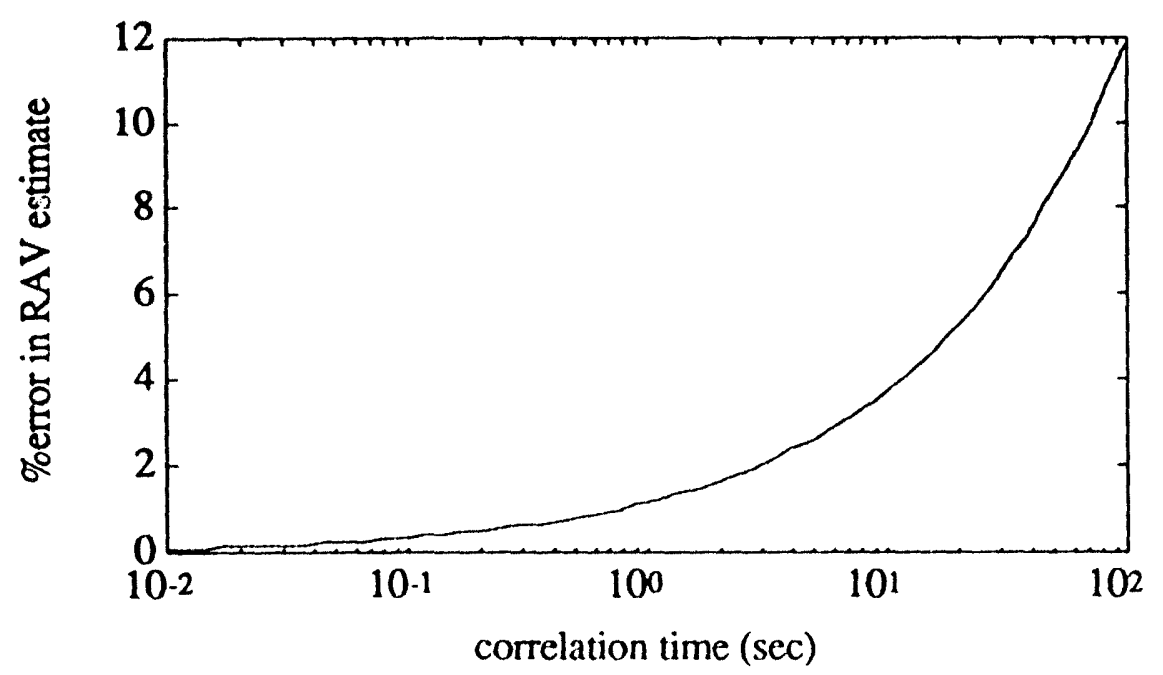

Figure $7 \%$ error in root Allan variance estimateusing one hour of data

For completeness, we fitted the data for the other axes as well. The least mean squares fitted Allan variance results are shown in Figure 8. The extraction of error terms are summarized in Table 5. Two observations can be made: (1) all axes meet the design specifications, and (2) the z-axis has a significantly higher (a factor of 10) quantization (or readout) noise than the other axes. The fact that the $z$-axis always appears to be more noisy is confirmed with previous analysis and observations $[10\}$. 


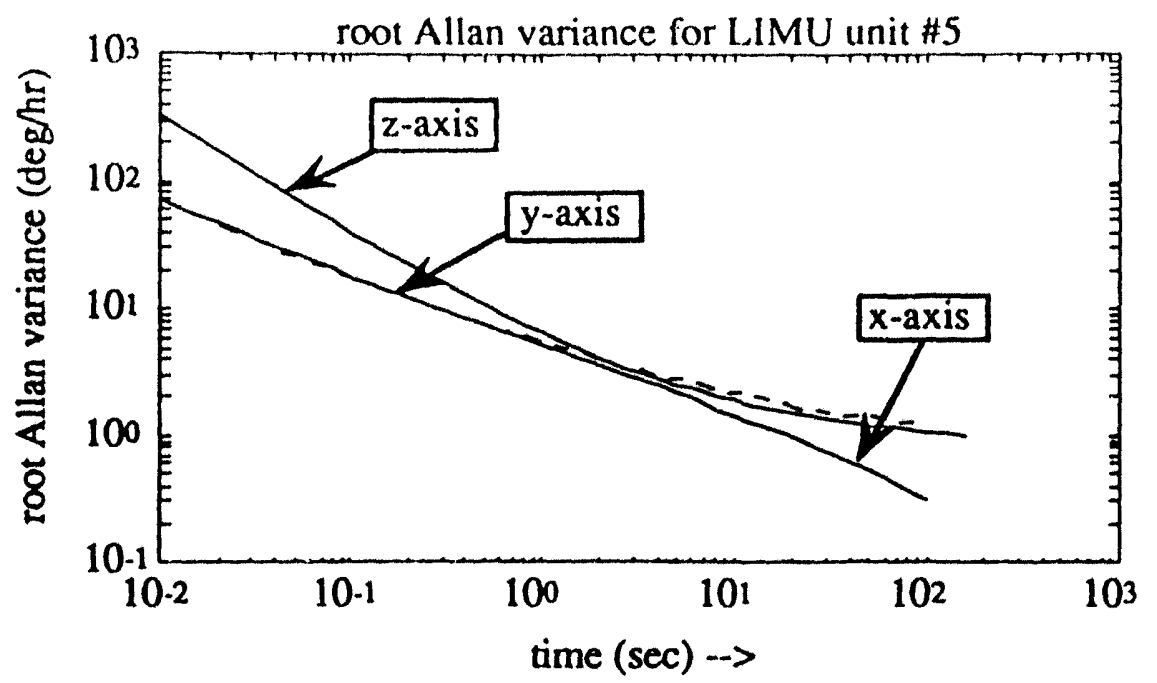

Figure 8 Comparison of root Allan variance estimates for $\mathrm{x}, \mathrm{y} \& \mathrm{z}$ axes

\begin{tabular}{|l|l|l|l|l|}
\hline & x-axis & y-axis & z-axis & $\begin{array}{c}\text { LIMU } \\
\text { Specification }\end{array}$ \\
\hline Quantization & $0.5 \mu \mathrm{rad}$ & $0.729 \mu \mathrm{rad}$ & $8.6 \mu \mathrm{rad}$ & $7.5 \mu \mathrm{rad}$ \\
\hline ARW & $0.08 \mathrm{deg} / \sqrt{\mathrm{hr}}$ & $0.074 \mathrm{deg} / \mathrm{hr}$ & $0.04 \mathrm{deg} / \mathrm{hr}$ & $0.125 \mathrm{deg} / \sqrt{\mathrm{hr}}$ \\
\hline Bias Instability & $0.14 \mathrm{deg} / \mathrm{hr}$ & $0.5 \mathrm{deg} / \mathrm{hr}$ & $0.52 \mathrm{deg} / \mathrm{hr}$ & $1 \mathrm{deg} / \mathrm{hr}$ \\
\hline
\end{tabular}

Table 5 Summary of LIMU performance for unit \#5

\section{Recursive Computation}

A recursive approach of root Allan variance computation was also implemented. Using the same set of data, we computed the quantization noise, angle random walk, and the bias instability. The results are summarized in Figures 9,10 and 11. Note the advantage of recursive implementation, it provides a convergence time history of the parameters of interest in addition to its real time computational capability. For example, the quantization noise converged in about 10 seconds, the angle random walk converged in about 2 minutes, and the bias term took as much as one hour to reach within $10 \%$ of its final value. 


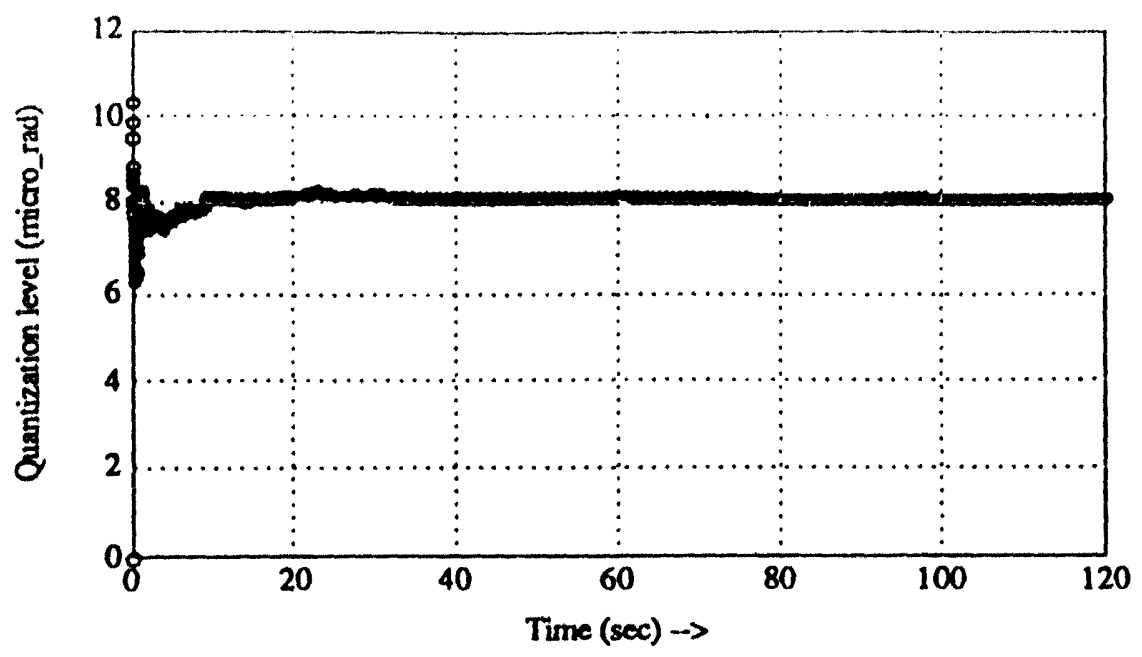

Figure 9 Recursive computation of quantization noise

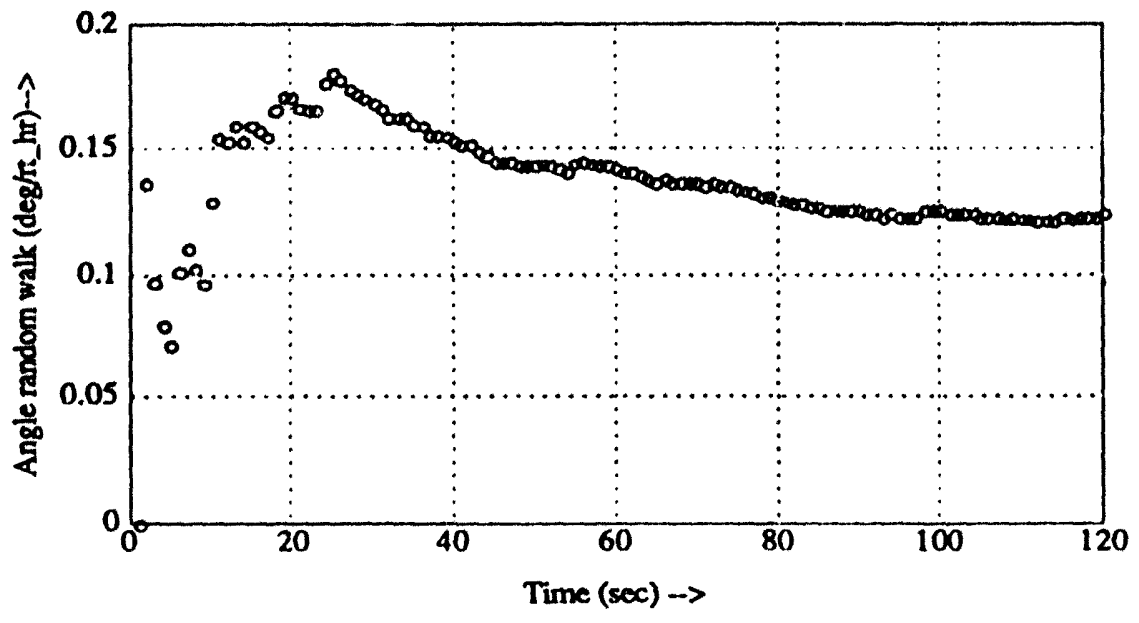

Figure 10 Recursive computation of angle random walk

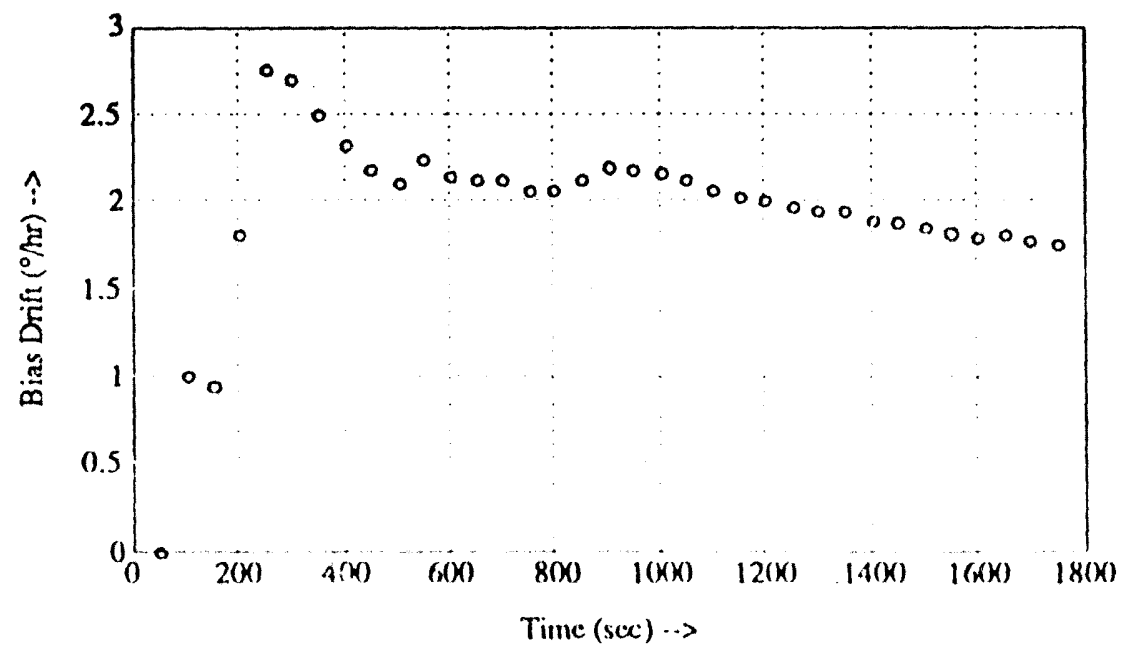

Figure 11 Recursive computation of bias instability 
Power Spectral Density and Auto-correlation

In order to show the difficulty in extracting individual error sources from conventional means such as PSD and auto-correlation, we present a small beginning segment of the the gyro output, its auto-correlation function, and the power spectral density respectively in Figures 12 , 13, and 14. Note that the correlation function has a correlation time (first zero crossing) less than 0.02 seconds. No significant correlation energy longer than 0.02 seconds or correspondingly no significant energy below $50 \mathrm{~Hz}$ in the PSD is present. Also note that correlation function peaks at $8.2 \times 10^{4}(\mathrm{deg} / \mathrm{hr})^{2}$ or $-286 \mathrm{deg} / \mathrm{hr} \mathrm{rms}$ noise which is close to the $300 \mathrm{deg} / \mathrm{hr}$ root Allan variance evalued at 0.01 seconds (see Figure 5). The different maybe the result of using only 30 minutes of data for the auto-correlation function and one hour of data for the root Allan variance computation.

Figure 14 shows the PSD plot. Note that components of error sources such as quantization, bias instability can be identified. However their true levels are difficult to extract - specially the angle random walk component - the primary error source of interest for specifying RLG performance.

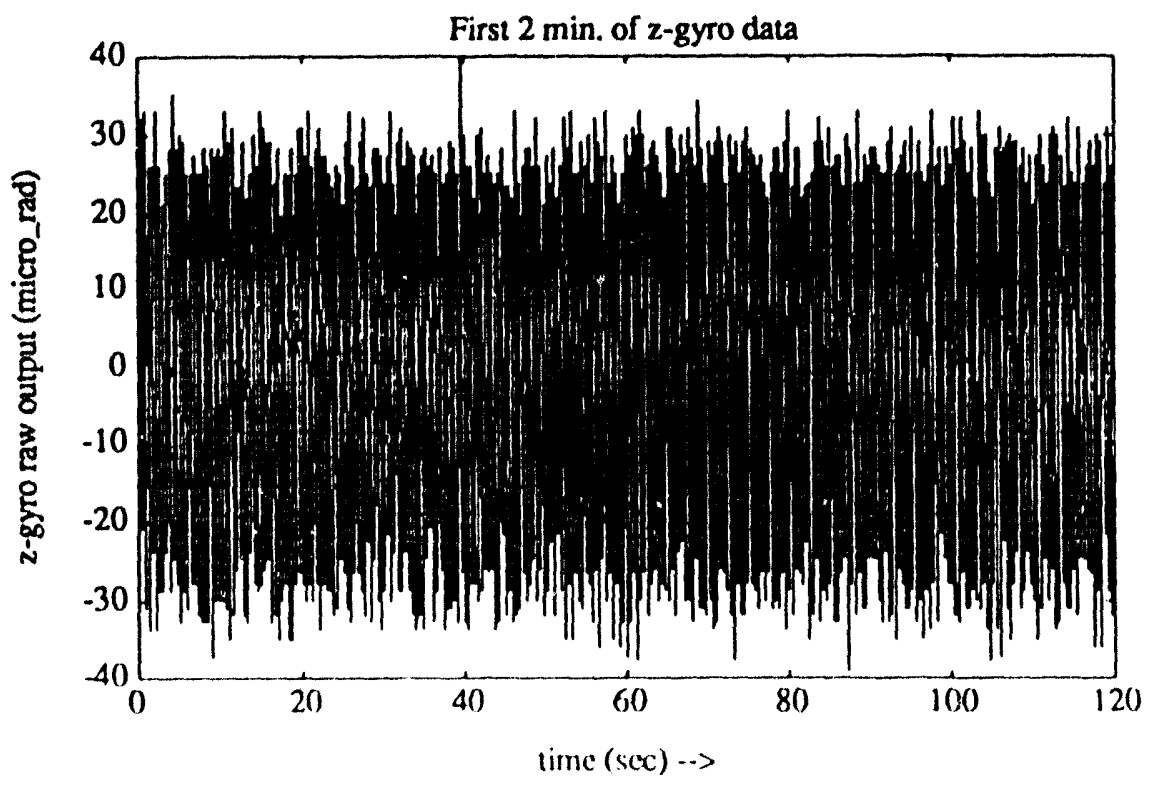

Figure 12 First 2 minute of $z$-gyro output 


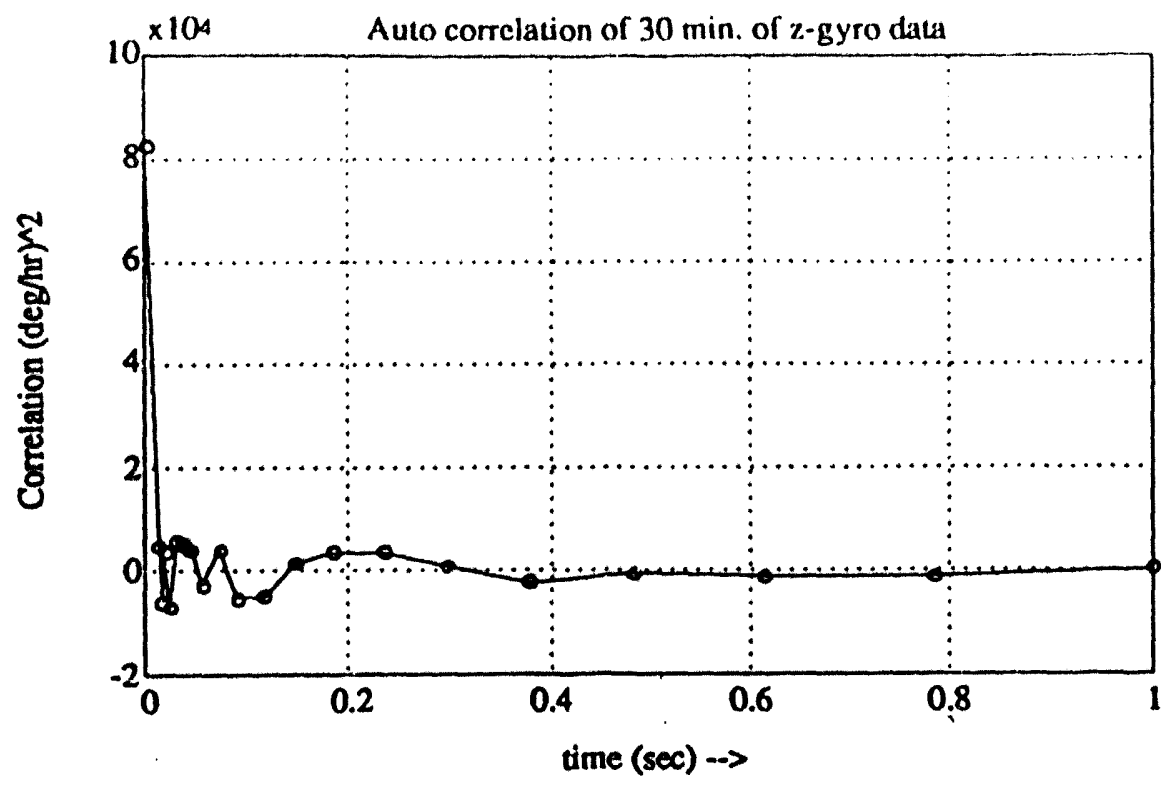

Figure 13 Auto-correlation function of 30 minute z-gyro data

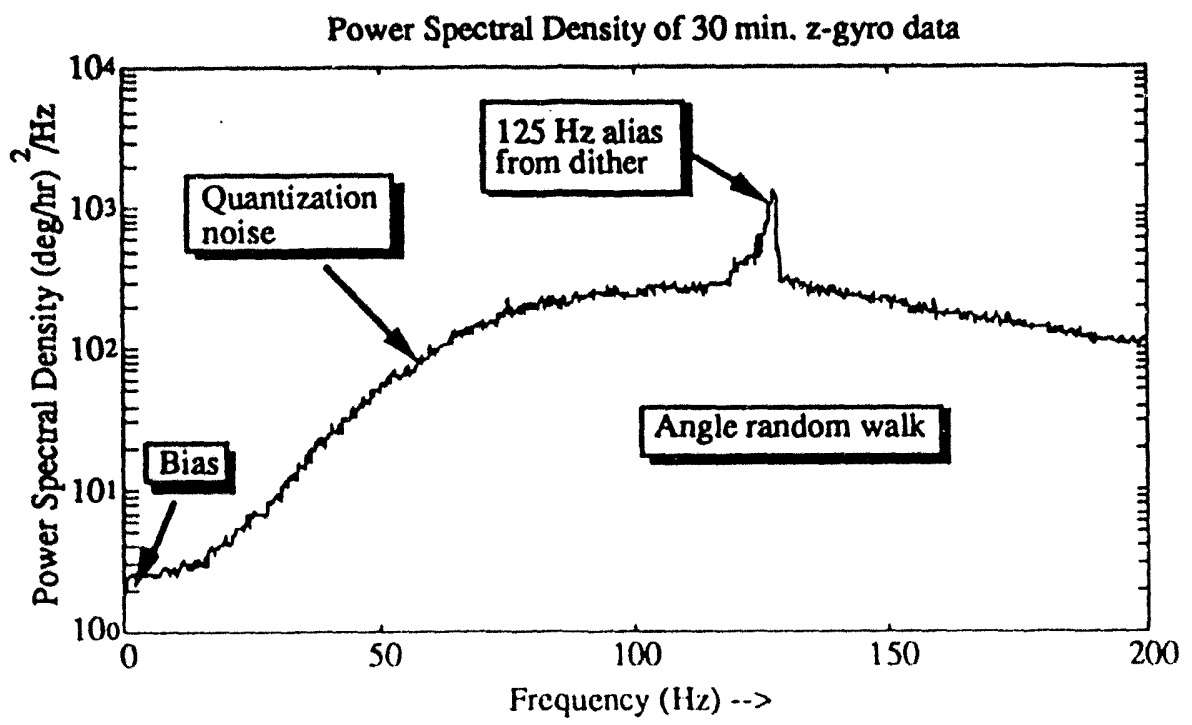

Figure 14 Power Spectral Density of 30 minute z-gyro data 


\section{L.IMU Performance Under Random Vibration}

A two minute sample of LIMU unit \#6 data was selected to estimate the noise error behavior under a dynamic environment. A random vibration excitation with an acceleration of $6.5 \mathrm{~g} \mathrm{rms}$ over a $2 \mathrm{kHz}$ bandwidth was applied to unit \#6. Angle random walk and quantization noise components were computed using recursive Allan variance formulation. The same computation was applied to a two minute sample of data from the same unit collected under a stationary laboratory environment. The results for the z-axis gyro are shown in Figures 15 and 16. Note that the results seem to indicate that unit \#6's gyros is insensitive to dynamic excitation.

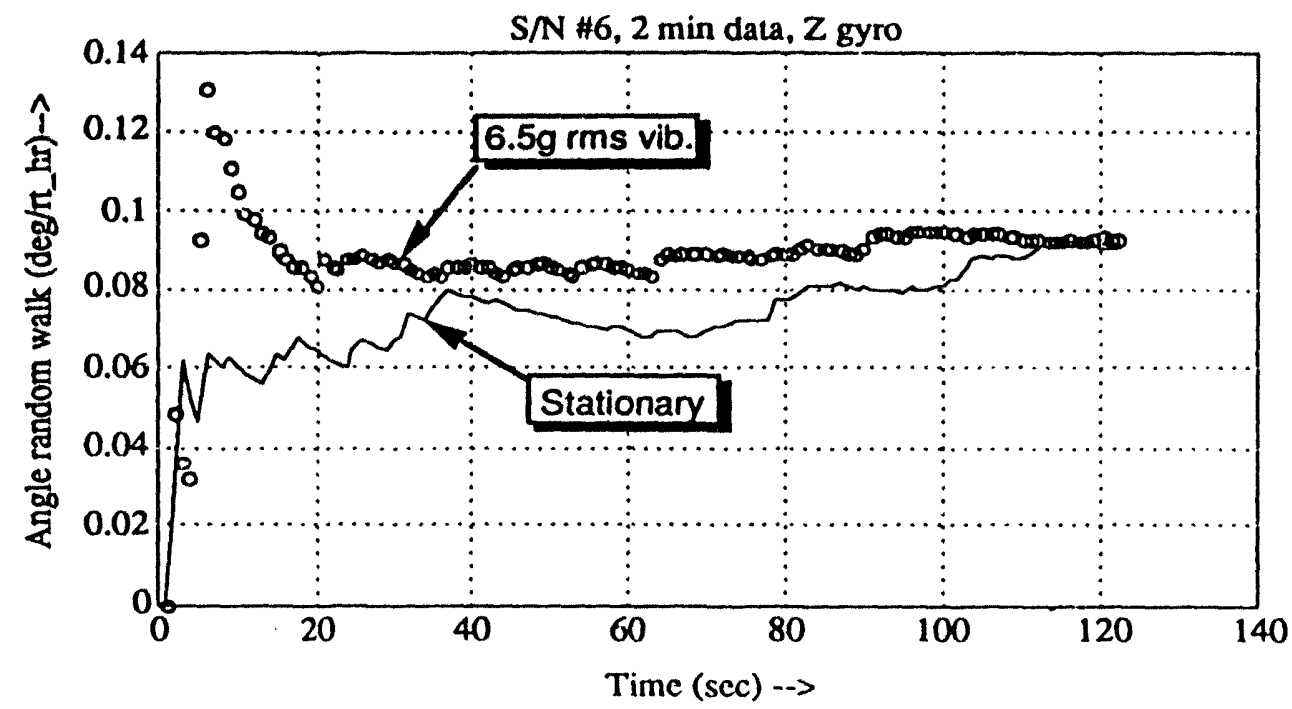

Figure 15 Angle random walk measured for LIMU unit \#6 z-axis

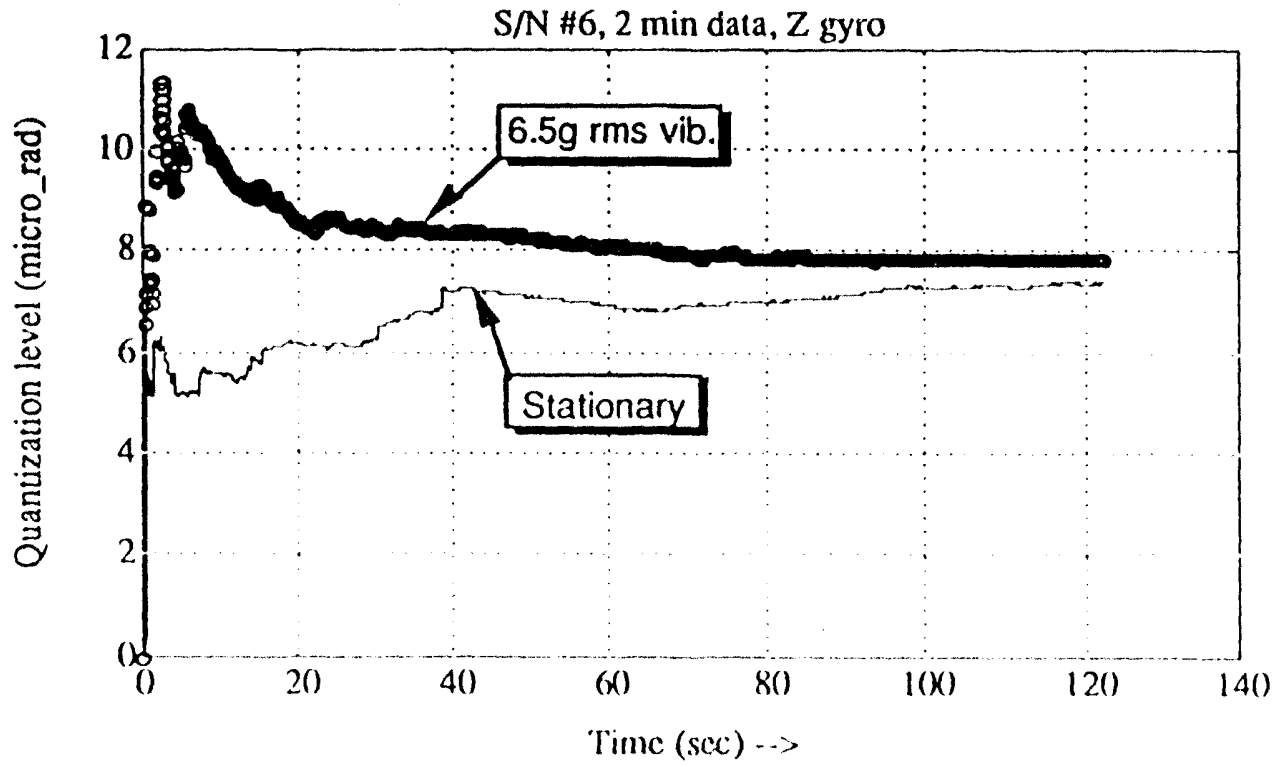

Figure 16 Quantization noise measured for LIMU unit \#6 z-axis 


\section{SUMMARY AND CONCLUSIONS}

This report summarized and explained the method of Allan variance for analysing the Ring Laser Gyro performance in terms of various error sources. These included quantization noise, angle random walk, bias instabililty, sinusoidal, rate random walk, and rate ramp. We presented two numerical approaches to compute Allan variance: batch implementation and recursive implementation. We also demonstrated how various error sources can be extracted from the Allan variance computation.

Using both implementations, we analysed several data sets collected from Honeywell LIMU units \#5 and \#6 based on the Allan variance approach. The purpose of this report is to illustrate the method of Allan variance computation and not to provide extensive analysis of LIMU performance. Detailed LIMU performance analyses will be provided by the IMU team [11]. However preliminary analysis reported here does show that LIMU gyros meet their design specifications including operation under a random vibration environment.

\section{Acknowledgments}

LIMU data used for illustration of Allan variance computation was supplied by Bart Beeman. Also the method of Allan variance for RLG performance analysis was first brought to my attention by Bob Steinkraus. Their cooperation and collaboration in making this effort successful is appreciated. 


\section{REFERENCES}

1. IEEE Standards Publications 647-1981," IEEE Specification Format Guide and Test Procedure for Single-Axis Laser Gyros (ANSI)."

2. Allan, David W.,"Statistics of Atomic Frequency Standards," Proceeding of The IEEE, Vol. 54, No.2, p. 221-230, February 1966.

3. Tehrani, M.M.,"Ring Laser Gyro Data Analysis with Cluster Sampling Technique," Proc. SPIE, Vol. 412, p. 207-222, 1983.

4. Papoulis, A., Probability, Random Variables, and Stochastic Processes. Third Edition, McGraw-Hill, Inc., 1991.

5. Killpatrick. J.,"The Laser Gyro," IEEE Spectrum, p. 44-55, October 1967.

6. Halford, D.,"A General Mechanical Model for If $\alpha$ Spectral Density Random Noise with Special Reference to Flicker Noise 1/ffl," Proceedings of IEEE., Vol. 56, No. 3, p. 251-258, March 1968.

7. Pasik, D.J. and Gneses, M.I., "A Ring Laser Gyro Strapdown Inertial Navigation System: Performance Analysis and Test Results," AIAA Guidance and Control Conference, Boston, MA., AIAA Paper No. 75-1095, August 20-22, 1975.

8. Save P.G.,"Laser Gyros in Strapdown Inertial Navigation Systems," Strapdown Inertial iNavigation Lecture Notes, Strapdown Associates, Inc., February 1990.

9. Morgan, A.A., "Static Data Collection for ACAL," Honeywell Interoffice Correspondence, Space Systems Group, Clearwater, Fl. March 4, 1991.

10. Vanrell, A.," Honeywell LIMU Noise Characteristics," Coleman Research Memo. No. CLM 93-116, May 18, 1993.

11. B. Steinkraus, B. Mathers, and B. Beeman, "LIMU Spin Test," LLNL Internal memorandum, IMU-01-93, August 31, 1993. 


\section{APPENDIX A}

\section{Derivation of Recursive Formulation of Allan Variance}

The computation of Allan variance is carried out in two steps: First, compute the average rate for each cluster using Eq. (A-1); and Second, compute the Allan variance from the rate averages using Eq. (A-2). Both of these equations can be recast into recursive forms whereby the computation can be carried out as soon as a new sample is arrived. Note that correlation time, $\tau_{M}$, and cluster length, $M$, is related by the equation, $t_{M}=M / f_{S}$, where $f_{S}$ is the data sampling frequency.

$$
\begin{aligned}
\varpi_{k}(M) & =\frac{1}{M} \sum_{i=1}^{M} \omega_{(k-1) M+i} \quad ; \quad k=1, \ldots K \\
\sigma_{A}^{2}\left(\tau_{M}\right) & \equiv \frac{1}{2}\left\langle\left(\varpi_{k+1}(M)-\varpi_{k}(M)\right)^{2}\right\rangle \\
& \cong \frac{1}{2(K-1)} \sum_{k=1}^{K-1}\left(\varpi_{k+1}(M)-\varpi_{k}(M)\right)^{2}
\end{aligned}
$$

We shall call the recursive rate formulation the rate recursion equation. It can be obtained readily as follow. Using (A-1) and consider the computation of the first cluster ( $M$ sample long) average rate by successive averages of cluster length $m$ with $1 \leq \mathrm{m} \leq \mathrm{M}$. Equation $(\mathrm{A}-1)$ can be rewrtten as follow:

$$
\begin{aligned}
\varpi(m) & =\frac{1}{m} \sum_{i}^{m} \omega_{i} \\
& =\frac{\omega_{m}}{m}+\left(\frac{m-1}{m}\right)\left(\frac{1}{m-1}\right) \sum_{i}^{m-1} \omega_{i} \\
& =\left(\frac{m-1}{m}\right) \varpi(m-1)+\left(\frac{1}{m}\right) \omega_{m} ; \varpi(0)=0 .
\end{aligned}
$$

Note that when $m$ reaches $M$, the cluster average rate $\Phi(M)$ is output immediately for computation of Allan variance using the recursive formulation of Eq. (A-2) - cluster recursion. Eq. (A-3) is applied for the first and each subsequent computation of cluster averages. 
Similarly the cluster recursion equation can be developed using (A-2) as follow:

$$
\begin{aligned}
\sigma_{K}^{2}(m) \equiv & \frac{1}{2(K-1)} \sum_{k=1}^{K-1}\left(\varpi_{k+1}(m)-\varpi_{k}(m)\right)^{2} \\
= & \left(\frac{1}{2(K-1)}\right)\left(\varpi_{k}(m)-\varpi_{K-1}(m)\right)^{2} \\
& +\left(\frac{K-2}{K-1}\right)\left(\frac{1}{2(K-2)}\right) \sum_{k=1}^{K-2}\left(\Phi_{k+1}(m)-\varpi_{k}(m)\right)^{2} \\
= & \left(\frac{K-2}{K-1}\right) \sigma_{K-1}^{2}(m)+\left(\frac{1}{2(K-1)}\right)\left(\varpi_{K}(m)-\varpi_{K-1}(m)\right)^{2}
\end{aligned}
$$

Note that Eq. (A-4) is valid for $\mathrm{K}>1$, and with $\sigma_{1}^{2}(m)=0$ as the initial condition. 


\section{APPENDIX B \\ Derivation of Frequency Domain Equivalence of Allan Variance}

We want to establish the equivalent relation between Allan variance and the two sided rate noise power spectral density (PSD) as given in Eq.(B-1). Note that a mathematical derivation was originally given in Ref. 2 \& 3. However a more detailed derivation is presented here for completeness and ease of access. The mathematical equivalent is given by the expression:

$$
\sigma^{2}(\tau)=\frac{1}{2}\left\langle\varpi_{k+1}-\varpi_{k}\right\rangle^{2}=4 \int_{0}^{\infty} d f S_{\omega}(f) \frac{\sin ^{4}(\pi f \tau)}{(\pi f \tau)^{2}}
$$

where $<>$ denotes the expected average, $\tau$ denotes the cluster length, and $S_{\omega}(f)$ is the angular rate noise PSD in units i Hertz. Now let $\omega(t)$ be the continuous gyro output waveform, then the average rate for the kth cluster can be written as:

$$
\begin{aligned}
\sigma_{k} & =\frac{1}{M} \sum_{i}^{M} \omega_{(k-1) M+i} \\
& =\frac{1}{\tau} \int_{t_{k}}^{t_{k}+\tau} \omega(t) d t,
\end{aligned}
$$

and the two sample variance can be expanded into:

$$
\sigma^{2}(\tau)=\frac{1}{2}\left\{\left\langle\varpi_{k+1}^{2}\right\rangle-2\left\langle\omega_{k+1} \varpi_{k}\right\rangle+\left\langle\varpi_{k}^{2}\right\rangle\right\}
$$

Now,

$$
\begin{aligned}
\left\langle\varpi_{k}^{2}\right\rangle & =\frac{1}{\tau^{2}} \int_{t_{k}}^{t_{4}+\tau} d t \int_{t_{k}}^{t_{t^{+}}+\tau} d t^{\prime}\left\langle\omega(t) \omega\left(t^{\prime}\right)\right\rangle \\
& =\frac{1}{\tau^{2}} \int_{t_{4}}^{t_{4}+\tau} d t \int_{t_{4}}^{t_{4}+\tau} d t^{\prime} R_{\omega}\left(t-t^{\prime}\right),
\end{aligned}
$$

where $\mathrm{R}_{\omega}$ ( ) is the autocorrelation function. But in general the autocorrelation function and the power spectrum is related by the Fourier transform: 


$$
R_{\omega}(\tau)=\int_{-\infty}^{\infty} S_{\omega}(f) e^{j 2 \pi f \tau} d f
$$

Therefore substituting (B-5) into (B-4) yields

$$
\begin{aligned}
\left\langle\sigma_{k}^{2}\right\rangle & =\frac{1}{\tau^{2}} \int_{t_{k}}^{t_{k}+\tau} d t \int_{t_{k}}^{t_{4}+\tau} d t^{\prime} \int_{-\infty}^{\infty} S_{\omega}(f) e^{j 2 \pi\left(t-t^{\prime}\right)} d f \\
& =\frac{1}{\tau^{2}} \int_{-\infty}^{\infty} d f S_{\omega}(f) \frac{\sin ^{2} \pi f \tau}{(\pi f)^{2}} \\
& =\int_{-\infty}^{\infty} d f S_{\omega}(f)\left[\frac{\sin ^{2} \pi f \tau}{(\pi f \tau)^{2}}\right]
\end{aligned}
$$

Furthermore using (B-6) in (B-4), we obtain:

$$
\begin{aligned}
\sigma^{2}(\tau) & =\frac{1}{2}\left\{\left\langle\Phi_{k+1}^{2}\right\rangle+\left\langle\varpi_{k}^{2}\right\rangle\right\}-\left\langle\varpi_{k+1} \varpi_{k}\right\rangle \\
& =\int_{-\infty}^{\infty} d f S_{\omega}(f)\left[\frac{\sin ^{2} \pi f \tau}{(\pi f \tau)^{2}}\right]-\int_{-\infty}^{\infty} d f S_{\omega}(f)\left[\frac{\sin ^{2} \pi f \tau}{(\pi f \tau)^{2}}\right] e^{j 2 \pi f \tau} \\
& =\int_{-\infty}^{\infty} d f S_{\omega}(f)\left[\frac{\sin ^{2} \pi f \tau}{(\pi f \tau)^{2}}\right]\left(1-e^{j 2 \pi f \tau}\right) .
\end{aligned}
$$

However the last factor in the above equation can be expanded as follow:

$$
\begin{aligned}
1-e^{j 2 \pi f \tau} & =1-\left[e^{j \pi \tau \tau}\right]^{2} \\
& =2 \sin ^{2} \pi f \tau-j 2 \sin \pi f \tau \cos \pi f \tau .
\end{aligned}
$$

Thus putting A-8 into A-7 and simplifying, we obtain the desired relation:

$$
\begin{aligned}
\sigma^{2}(\tau) & =2 \int_{-\infty}^{\infty} d f S_{\omega}(f)\left[\frac{\sin ^{4} \pi f \tau}{(\pi f \tau)^{2}}\right]-j \int_{-\infty}^{\infty} d f S_{\omega}(f) \cos (\pi f \tau)\left[\frac{\sin ^{3} \pi f \tau}{(\pi f \tau)^{2}}\right] \\
& =4 \int_{0}^{\infty} d f S_{\omega}(f)\left[\frac{\sin ^{4} \pi f \tau}{(\pi f \tau)^{2}}\right]
\end{aligned}
$$

Note that the second term vanishes because the power spectrum, $S_{\omega}(f)$, is an even function for a real data sequence. 
AIT-IGC-93-003

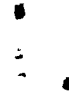



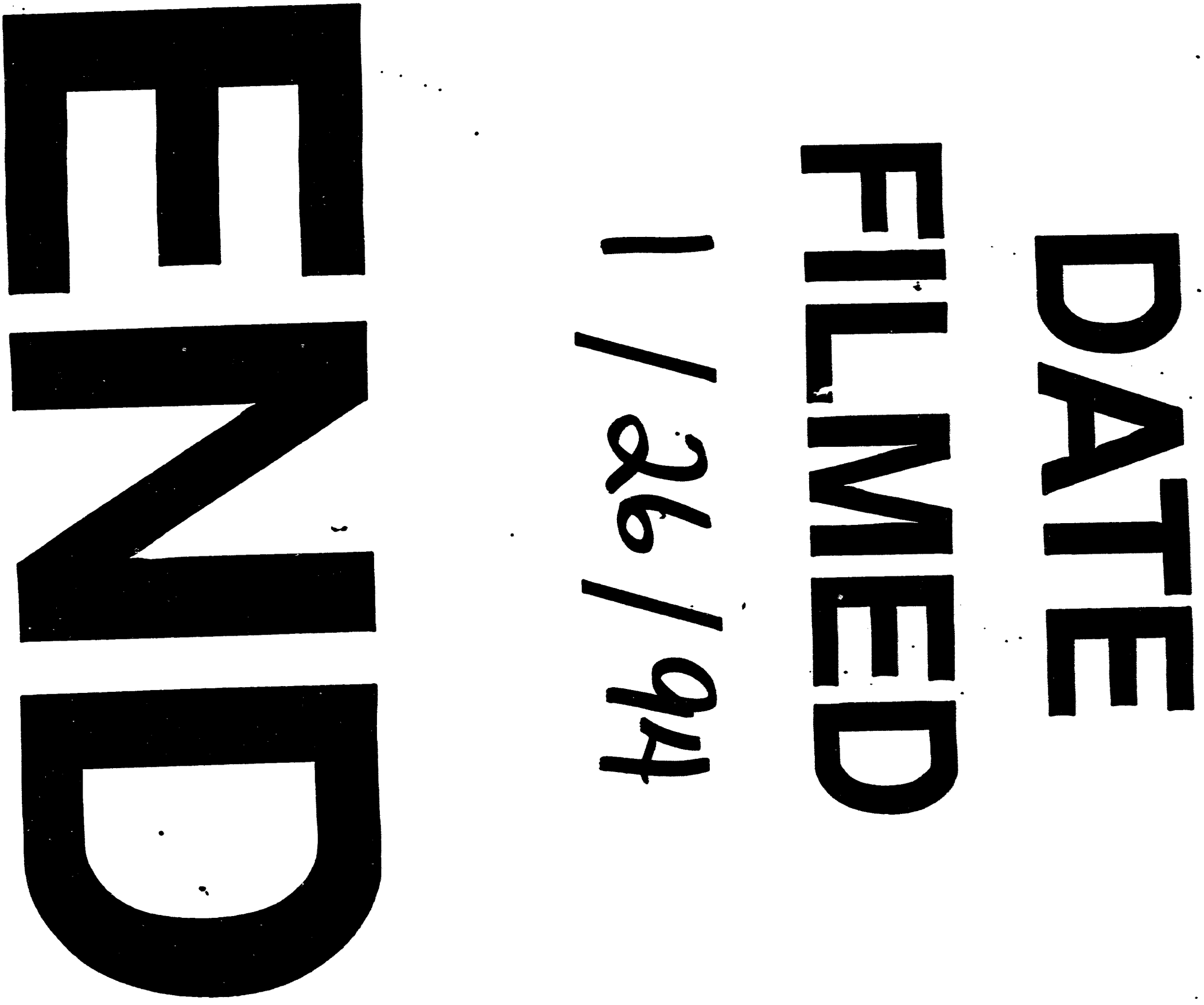

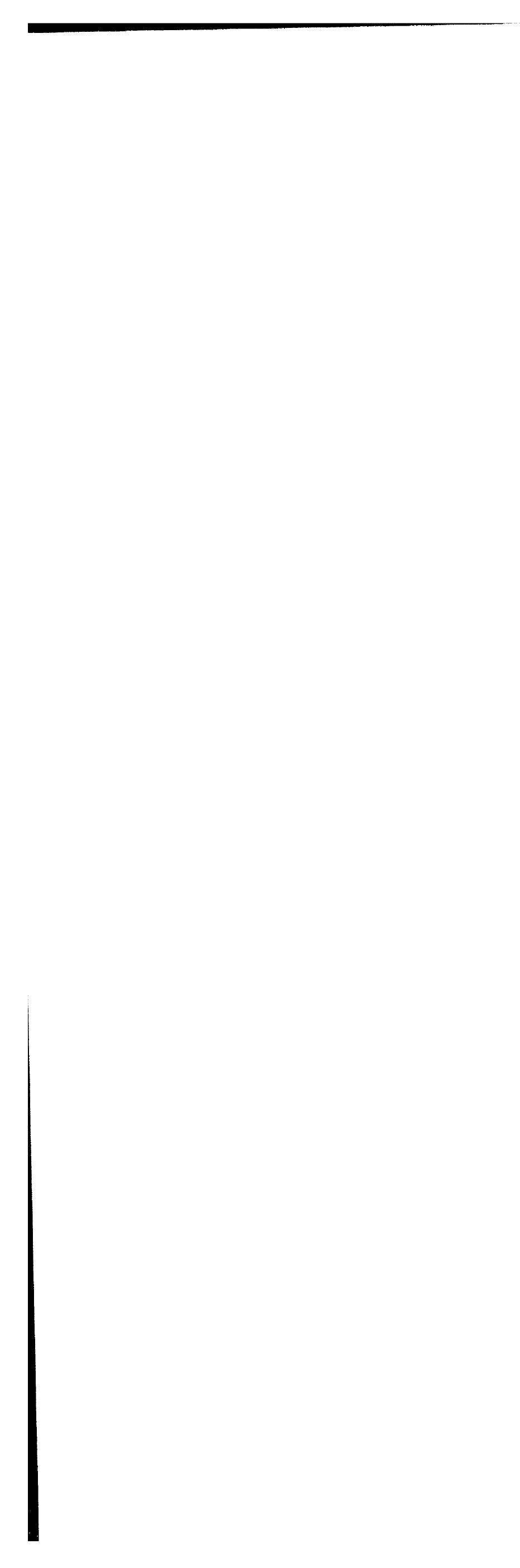

(1)

(5) 